\title{
Expert Panel Discusses the Importance of Systems Medicine
}

\author{
Moderator: Harald H.H.W. Schmidt, ${ }^{1, *}$ \\ Participants: Jan Baumbach, ${ }^{2, *}$ Joseph Loscalzo, ${ }^{3,4}$ Alvar Agusti, ${ }^{5-8}$ Edwin K. Silverman, ${ }^{9,10}$ and Vasco Azevedo ${ }^{11}$
}

Dr. Harald H.H.W. Schmidt: We have the opportunity to make the just launched Systems Medicine journal the flagship of our new discipline. Thanks to our panelists and readers for joining in.

I brainstormed a bit on the key questions in our field. One would be, "What is systems medicine, or network medicine, in comparison to systems biology?" Is it just systems biology but only in one species? Probably not.

Then a couple of others: Why does systems medicine matter? What is its potential impact? Are there risks? What are the gaps at the moment? And what are the next steps? These are some of the questions that I suggest we address.

So perhaps we start with the first one: What is systems medicine or network medicine?

Dr. Joseph Loscalzo: Network medicine or systems medicine reflects the marriage of systems biology and network science in an effort to understand the complexity of the interactions among all the molecular determinants of a disease phenotype.

In this respect, one can conceive of the universe of molecular data sets that contribute to phenotype, either normal or disease phenotype, in the usual omics subset-from the genome to the transcriptome to the proteome to the metabolome and then to the physiome or phenome. Each of the subsets can be modified in different ways by environmental exposures, such as stochastic post-translational modification of the proteome, or epigenetic changes in gene expression that are environmentally driven.

As one thinks through these layers, one can imagine that they comprise a complex network of networks that have various levels of interaction that ultimately drive the phenotype. One can also consider that any of these systems or networks is a means by which molecular information flows through the system.

The dynamics of that flow of molecular information, and the structures and their modifications that accompany perturbation, either through genetic variation or through environmental perturbations, really account for the differences between normal or disease phenotype. Normal systems or networks underlie human biology, or health and dysfunctional networks underlie human pathobiology or disease.

Dr. Alvar Agusti: I fully agree with Joe, but I would like to make some additional comments. I think that there are two key words here. One is complexity, and clearly biology is complex. No question. And the other is integration because that is the only way to try to grasp all these complexities.

As clinicians, we are trained precisely to integrate things-symptoms, physical findings, blood tests,

\footnotetext{
${ }^{1}$ Department of Pharmacology \& Personalized Medicine, Faculty of Health, Medicine \& Life Science, Maastricht University, Maastricht, the Netherlands.

${ }^{2}$ Department of Bioinformatics, Technical University of Munich, Munich, Germany.

${ }^{3}$ Hersey Professor of Theory and Practice of Medicine, Harvard Medical School, Harvard University, Boston, Massachusetts.

${ }^{4}$ Department of Medicine, Brigham and Women's Hospital, Boston, Massachusetts.

${ }^{5}$ Respiratory Institute, Hospital Clinic in Barcelona, Barcelona, Spain.

${ }^{6}$ Department of Medicine, University of Barcelona, Barcelona, Spain.

${ }^{7}$ Institut d'investigacions biomediques August Pi I Sunyer (IDBAPS), Barcelona, Spain.

${ }^{8}$ Centro de Investigación Biomédica en Red (CIBER) de Enfermedades Respiratorias, Spain.

${ }^{9}$ Channing Division of Network Medicine, Brigham and Women's Hospital, Boston, Massachusetts.

${ }^{10}$ Department of Medicine, Harvard Medical School, Boston, Massachusetts.

${ }^{11}$ Federal University of Minas Gerais, Belo Horizonte, Brazil.

${ }^{*}$ Co-Editors-in-Chief.
}

(c) Harald H.H.W. Schmidt et al. 2018; Published by Mary Ann Liebert, Inc. This Open Access article is distributed under the terms of the Creative Commons License (http://creativecommons.org/licenses/by/4.0), which permits unrestricted use, distribution, and reproduction in any medium, provided the original work is properly cited. 
imaging, lung function, etc. The problem with the biological complexity is that the human brain has a limit in its capacity to integrate information, so now we need bioinformatics and network analysis.

Dr. Loscalzo: Yes, Alvar, I agree that in an odd way we, as physicians, are used to looking at patients in a bit more integrative or holistic way as we think about the constellation of symptoms, signs, molecular markers, and other abnormal or normal laboratory tests with which they present. Almost all of what we do is not based on a deep understanding of the molecular pathways. It is basically grounded in experience and based on our intuition and pattern recognition.

In this respect, the complexity of the interactions that drive phenotypes of interest requires that we understand precisely how these systems are controlled, and is what systems or network medicine affords such an approach. This discipline advances our skills up several orders of magnitude as diagnosticians and precision medicine therapists if we understand the network layout and the drivers of disease.

Dr. Agusti: I agree, Joe. But sometimes people ask me, "What is the difference between systems medicine and traditional physiology?" I believe that the main difference is the level of molecular understanding, as you said, because integrative physiology is also about integration, and this is what we use every single day in clinical practice.

Dr. Jan Baumbach: What is, then, the difference between systems medicine and systems biology?

Dr. Agusti: To me, the main difference between systems biology and systems medicine (or network medicine) is that the former deals with preclinical models and organisms, whereas the latter deals with human beings and clinical problems. This difference has important consequences. First, nonhuman systems are easier to manipulate and intervene. Second, not all findings in nonhuman systems are directly applicable to humans.

Dr. Edwin Silverman: I agree with that insight. What I am especially excited about with the field of network medicine, in general, and the Systems Medicine journal, in particular, is the application of these new molecular technologies to complex diseases. There has certainly been a lot of excitement, which I share, about the potential of complex disease genetics to provide new insights into disease pathogenesis. However, given the small effects of the vast majority of complex disease genetic determinants, genetics is unlikely to be really transformative in medical care of complex diseases. I think it is going to be the use of genetics in conjunction with other omics technologies in a network context that could really give us new biomarkers of disease diagnosis, understanding of disease heterogeneity, and accurate prediction of disease progression.

Dr. Baumbach: I totally agree. As a bioinformatician and a computational biomedicine researcher, I definitely see a problem with respect to the complexities of diseases. We will probably have to rely on pattern recognition technology that goes way beyond what we as humans can achieve.

In other words, we put the future of ourselves, of patients, into the hand of a machine. And then we are reaching an ethical point, but we also reach a point where we lack proof of concept, at least on a broad scale.

Dr. Schmidt: On that note, perhaps we can discuss: What is the difference between systems medicine and current medicine? If I may start, one problem that classical medicine faces today is the lack of mechanistic understanding in almost every disease area, and where this has really hit the wall is in drug discovery. Since the '50s, the efficacy of drug discovery per time and money invested are constantly declining. One major contributor to this is the disease definitions that we are using, which are mainly based on organs, symptoms, or sometimes just on the name of a doctor, Alzheimer, Parkinson, and so forth, which really are not molecular disease definitions.

In hypertension, for instance, $\mathbf{9 5 \%}$ of the patients are diagnosed with essential hypertension, which means you have elevated blood pressure, certainly a risk factor, but we do not know why. And then patients are treated with drugs that dilate blood vessels. Of course, the symptom, elevated blood pressure, disappears, but the cause of disease is not treated because we do not know it. That is just one example. Also the way we divide medicine up into different disciplines and different clinics, basically one clinic per organ. That is probably not sustainable.

I think what systems medicine eventually can help physicians do is to regain this holistic view of their patients, but not in an intuitive manner as today, but in a very data-based one-they get something measurable, definable, understanding comorbidities, 
identifying molecular mechanisms and suggesting effective treatment in a mechanism-based approach.

Dr. Silverman: I agree with that completely, and I think the current structure of healthcare delivery also limits our ability to bring advances in quantitative biomedical research, computational biology, and complex disease genetics effectively into clinical care.

If we recognize that complex diseases such as diabetes, asthma, and coronary heart disease are really heterogeneous syndromes that are influenced by genetic and environmental factors within a developmental context, systems medicine approaches have the potential, at least, to dissect those complex syndromes into biologically based subtypes and give us new biomarkers that would be useful for disease diagnosis, assessment of progression, and response to treatment.

Dr. Schmidt: Probably genetics can achieve much more, but not when you perform genetics on illdefined disease phenotypes. I know Alvar [Augusti] had a very nice article recently on splitting up classical into molecularly defined endophenotypes.

Dr. Agusti: Thanks Harald. In that article, ${ }^{1}$ we argue that we need to understand much better the biological mechanisms (or endotypes) underlying most human diseases, particularly those of chronic nature. These endotypes are the end-result of gene-environment interactions through complex biological networks. So, I believe that there is a clear window of opportunity for research approaches, such as those of systems biology. However, I think that it is fair to recognize that any review on the topic should start by recognizing that medical practice in the past, let us say, 50 years has exploded. There are many examples now in cardiology, oncology, endocrinology, and more. Diseases are treated very differently and much better than when I finished my medical school 40 years ago. So, I think that yes, it is okay to recognize our limitations and to try to go beyond them, but at the same time, I think it is fair to recognize that clinical care today, at least in developed countries, is quite impressive in many, many circumstances.

Dr. Schmidt: So, to stay with my example with hypertension, it is of benefit sometimes to do symptomatic therapy, like to remove this risk factor of elevated blood pressure. That has real patient benefit. And therapies like that have certainly contributed to lowering of mortality or disease severity.
Forty years ago, these were low-hanging fruits from a drug development perspective, basically treating symptoms without understanding the molecular mechanism of disease. Now it is close to impossible to introduce such drugs. Thus true innovation is happening in only a few areas, that is, oncology, immune diseases, and antivirals, but everywhere else, there is hardly any conceptual progress and no new validated targets within those fields of medicine. So, we obviously need a major innovation. We need new mechanisms and new approaches. It is the potential of Systems Medicine to help closing this gap and unmet medical need.

Dr. Agusti: I fully agree. We need to go beyond that. There are many reviews, as you said, showing that no new molecules are coming to the market. That is absolutely true. However, life expectancy in humans has improved dramatically in the past 100 years. So even treating patients based on symptoms has produced an outstanding change in survival. There is still an opportunity to improve that.

Dr. Silverman: I am a bit ambivalent about this issue, because on the one hand, I do share Alvar's opinion that there have clearly been areas where clinical care has transformed dramatically since he and I were in medical school decades ago. For example, effective treatments of some types of previously lethal cancers are now available.

On the other hand, I still feel that there are so many common, complex chronic diseases wherein the treatments have not changed that much, and the outcomes are still quite suboptimal.

The hypertension example is an interesting one. It is a physiological biomarker in which many people are completely asymptomatic with their hypertension, but by identifying that biomarker, it was able to be used as a treatment target and lead to substantial reductions in cardiovascular disease morbidity and mortality.

I think the question now is: How can we take the vast amounts of omics data that are being generated and develop effective molecular biomarkers for the future using systems and network approaches?

\section{Dr. Schmidt: Mechanistic biomarkers.}

\section{Dr. Silverman: Exactly.}

Dr. Loscalzo: It is also useful to think about trajectoriestime trajectories in healthy aging, as well as in the 
transition from health to disease. I think there are many ways by which to define "normal," the evolution of normal health as a function of time. There is not a single trajectory in whatever combination of markers or whatever combination of networks one explores.

Obtaining more information about the range of normal in these dynamic systems will be very important. Equally important is getting a better sense of the predictors of the transition from health to disease for specific diseases. Some have viewed this process much as looking at a landscape in which one measures some kind of physical potential with stable wells in the landscape. There are points in that landscape in which one can identify the transition from a well of illness, with treatments pushing the person back or the system back toward a well of health. This is a simple metaphor, but the principle is that it will be important to obtain more information about the time-dependent changes in the transfer of molecular information across these complex networks in healthy states and disease states and in a transition from health to disease. Doing so can begin to give us more insight into how best to define useful molecular mechanistic biomarkers and how best to tailor not just therapies for established disease, but also preventive therapies or preemptive therapies in patients headed toward disease.

Dr. Schmidt: Thinking of prevention in that context brings us to the point, having now defined what systems medicine is, and what we see as its potential, maybe we should also think about what potential risks are, for example, of overpromising too much too soon, and what are next steps?

Dr. Baumbach: So, one substantial risk is a lack of data when we talk about following many people over time for constant measurement. Even now we often use case control studies, that is, simple study designs, and we usually sample at the extreme ends of the distribution, such that we have the highest likelihood of seeing a clear distinction between the two groups under investigation. But then we lack everything in the middle.

A disease, of course, is a progression over time with shifts in the molecular programs controlled by genes, proteins, and so on. However, before we can start modeling all this, we really need to get the data. So, I wonder whether where we are with (1) the amount of data that we havedo we have most of what we need? And (2) if we do, can we access it efficiently? And (3) access it ethically?

There are many genomics studies here in Denmark, for example, and we clearly see driver mutations for certain cancer types, but we are legally not allowed to contact the patient because cancer was not the study's focus. So, it is also an ethical and a legal problem.

I wonder where we stand with this. There are many problems and risks that need to be overcome.

Dr. Silverman: I think that the good news is that there are major efforts ongoing, such as the Trans-Omics for Precision Medicine (TOPMed) program from the National Heart, Lung, and Blood Institute of the U.S. National Institutes of Health (NIH), which are generating vast amounts of omics data. Whole genome sequences have been generated in more than 100,000 people by TOPMed; as the program moves forward, vast amounts of RNA-Seq data, proteomics data, and metabolomics data will be produced. I think that resources such as TOPMed have tremendous potential.

One challenge is that most of those omics measurements are not longitudinal. In addition, many of the available biospecimens have been collected after disease has already developed. So, it is difficult to create biomarkers for incident disease with currently available data. And the ability to incorporate environmental factors is still a major challenge in terms of how to assess them and how to integrate them into the analyses.

From my perspective, the big risks are not so much the availability of data, but coming up with more effective ways to analyze the data, and also being careful not to overpromise. I think that in some other fields, investigators have made the mistake of promising the world in a short period of time. We need to recognize that physicians are often averse to quantitatively based approaches. If we do not come up with some clear early wins in the diagnosis, prognosis, and treatment of complex diseases that can be used as guideposts for other diseases, there is going to be a lot of pushback.

And we also need better training. We need to bring those quantitative approaches into medical school and residency training for physicians of the future.

Dr. Agusti: Ed just took the words from my mouth. I think that training the next generation of clinicians in this field is absolutely key for medical school students, medical residents, and for medical researchers.

Dr. Schmidt: The U.S. NIH is clearly a strategic catalyst in this movement, but the European Union's (EU) funding initiatives are also shifting in medicine to in silico, big data, systems, and network approaches that will have clinical impact. So, from top down, I think the key 
players in research funding have clearly recognized that this is the way forward. It may be a good idea to hold joint workshops and brainstorming meetings with their representatives. We should make sure we get EU and NIH stakeholders and decision-makers on board. There are a couple of fantastic white articles that have been written at the NIH. ${ }^{2}$

But there is also another important aspect on big data, availability. In some countries, such as Germany, for instance, patients are worried about contributing their anonymized data for research applications. It will, therefore, require a lot and appropriate communication as these next advances in systems medicine can only be achieved when having access to anonymized data from hundreds of millions of patients. The advances that we are making now are usually based on those countries that were a bit more visionary early on: some of the Scandinavian countries, the United Kingdom, and others. But I think we need it on a much wider scale together with the appropriate data safety.

Dr. Baumbach: Well, as a bioinformatician and computer scientist, I can tell you. Of course, once some data are generated, it will probably never be forgotten. But in contrast, these data only make sense together with all the other metadata. Think about this scenario: Imagine you would have the option to pay, let us say, $\$ 20$ a month, and Google would just give you what you want-Google Maps, etc.,-but refrain from collecting meta-information from your device, such as your user behavior.

One may ask. "Why is Google not offering such a deal?" The reason is not just money. They simply cannot allow this $\$ 20$ buyout because the only reason why they can offer you their service is because everyone is contributing to this big data collection and big data mining platform. And it is similar here with systems medicine, right?

The data will be there, and there are different ways to protect it. But that is only one part of the story.

The other part is that this whole research field is still in its infancy. It should become a whole part of systems medicine, including data protection development. Maybe it is possible to develop methods allowing data to be analyzed in a distributed manner so that you can do big data analysis with software run on different servers in several hospitals, such that one does not need to centralize all analyses on one big server doing everything.

So there might be ways to distribute the risk in this sense as well, but as I said: The whole research field is just in the beginning.
Dr. Schmidt: Yes and the key thing is to anonymize the data.

Dr. Baumbach: That is just one aspect. I now live in Denmark where I could usually track down from only the genome data at least to the birth village of a sample donor and the donor's age. And there are many other ethical issues.

Imagine, for instance, a set of homozygote twins, and one twin is giving consent to having his or her data used and the other twin is not. But then the other twin has no choice because his or her genome is identical and the first twin decided to give his or her information away. So twin number 2 automatically gives his or her consent-whether he or she wants to do so or not. All these problems need to be overcome, and anonymization is only one major step.

\section{Dr. Schmidt: What are other next steps we need to take?}

Dr. Silverman: We need to recognize that even within computational biology fields, there are intellectual siloes that need to be broken down. How much do network scientists really communicate with complex disease geneticists, machine-learning experts, and researchers generating the omics data? We need to think about how we can overcome those barriers that do exist.

Then, we need to have ongoing efforts in methods development to figure out how to integrate multiple omics data most effectively and, as we mentioned, to include environmental factors.

Harald, you had spoken about drug development. How do we change drug development and treatment paradigms from focusing on one drug target to treating an entire syndrome, and to move from that classic model to focus on multiple drug targets potentially in a dynamic sequence in subsets of patients, rather than in a whole group of patients with a complex disease?

Dr. Schmidt: We should acknowledge that we are always treating subnetworks of the entire interactome when we treat a disease. And it is quite unlikely that you are able to modulate a subnetwork effectively that has slightly deranged into an unhealthy feature by one drug.

There are increasing examples that drugs are not just combined, but they are also mechanistically linked and synergized with each other. And that is definitely the future. 
As a pharmacologist, I will share the slightly provocative hypothesis that we probably have already, let us say, $80-90 \%$ of all the drugs that we need. We just need to apply them to the right patients and to the right disease phenotypes. Clever repurposing of existing drugs, maybe even so-called fallen angels-drugs that were tried to develop but were tested on patient cohorts who were not stratified well enough because the mechanism of the drug was not a stratification parameter-could also be resurrected.

We may not need so much drug discovery; maybe a lot of repurposing and clever targeting of drugs to the right patient will see a lot of early gains.

Dr. Agusti: I will add something with respect to what is next. It might seem very silly, but I think it is important. Certainly, there is a great opportunity, the idea of systems and network medicine is fantastic, but it is going to be difficult because the human body is enormously complex. It is not only about the complexity within one cell type. It is that there are many cell types, many organs, some of them interacting, and so on. The only way forward in this field, like in any other research field, is to work hard, share and discuss your science with colleagues, and go back to work again! No doubt we will find new challenges and new questions, but I am certain that, with time, human science will overcome them. It is a matter, to some extent, more trial and error and working hard, getting connected, sharing experiences, and trying to move the field forward. It is going to take time and a lot of work.

Dr. Schmidt: Also the communication of all of this into the medical community is quite important. So that we explain what we are actually doing. And when we are saying we want to redefine diseases and redefine disciplines that, at first glance, might sound a bit alarming to some specialists. I think communicating very well what systems medicine is about is key-that it is a kind of revolution but one that is well thought through and not to be perceived as something that is threatening. But there may be acceptance issues with some of our colleagues.

Dr. Loscalzo: One of the things that we can do through the Systems Medicine journal that might help with this education-because it does require a deep educational process-is to take some fundamental principles with which we all agree that offer both the promise and po- tential of systems or network medicine and pick a wellestablished, generally recently published observation that supports that principle. Such an example could be anything from using a network strategy to repurposing drugs or identifying a novel pathway that drives a disease phenotype only appreciated by a systems or network analysis.

The body of evidence supporting the utility and benefits of network medicine continues to grow, is doing so exponentially now, I am pleased to see. Thus, I think that this is one of those conceptually challenging areas, even for bright physician scientists, where illustrating principles by example is probably the best way to facilitate learning rather than through complex diagrams and equations.

Dr. Agusti: I think that is a good idea. I would suggest complementing it in the journal with a section wherein we can highlight articles published in other journals along these lines. Look, if you read our journal, you will also find samples that we picked from some much more specialized journals and make a comment or highlight what is important, what is relevant, or what might be useful.

\section{Dr. Loscalzo: Great idea.}

Dr. Schmidt: Yes. Let us do that. We will make our journal, Systems Medicine, attractive for medical specialists who are open to systems medicine-to really make it the primary place to look for any relevant information in this area. We will do exactly what you described, educate, and be a radar for what is happening in breakthroughs in systems medicine.

\section{References}

1. Agusti A, Celli B, Faner R. What does endotyping mean for treatment in chronic obstructive pulmonary disease? Lancet. 2017;390:980-987.

2. Boname ML, Wagner Gee A, Wizemann T, et al. Enabling precision medicine: the role of genetics in clinical drug development: proceedings of a workshop. 2017. www.nap.edu/24829, DOI: 10.17226/24829, Accessed November 22, 2017.

Cite this article as: Schmidt $\mathrm{H}$, Baumbach J, Loscalzo J, Agusti $\mathrm{A}$ Silverman EK, Azevedo V (2018) Expert panel discusses the importance of systems medicine, Systems Medicine 1:1, 3-8, DOI: 10.1089/sysm.2017.29000.rtd.

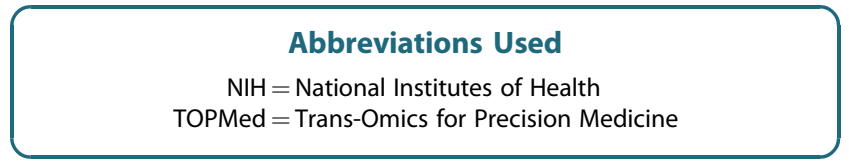

